\title{
VARIABILIDADE DO DESEMPENHO NO LINE-DRILL TEST EM ADOLESCENTES JOGADORES DE BASQUETEBOL
}

\author{
PERFORMANCE VARIABILITY IN LINE-DRILL TEST IN ADOLESCENT BASKETBALL PLAYERS
}

Artigo Original

Original ARTICLE

VARIABILIDAD DEL DESEMPEÑO EN EL LINE-DRILL TESTEN JUGADORES ADOLESCENTES DE BALONCESTO

Artículo Original

\author{
André Luiz de Almeida Soares \\ (Educador Físico) \\ Thiago José Leonardi 1,2,3 \\ (Educador Físico) \\ Riller Silva Reverdito 1,4 \\ (Educador Físico) \\ Carlos Eduardo Gonçalves ${ }^{5}$ \\ (Educador Físico) \\ Roberto Rodrigues Paes ${ }^{1}$ \\ (Educador Físico) \\ Humberto Moreira Carvalho' \\ (Educador Físico)
}

1. Faculdade de Educação Física, Universidade Estadual de Campinas (Unicamp), Campinas, SP, Brasil.

2. Faculdade Adventista de Hortolândia, Hortolandia, SP, Brasil.

3. Faculdade de Jaguariúna, Jaguariúna, SP, Brasil.

4. Faculdade de Ciências da Saúde, Universidade do Estado de Mato

Grosso, Cáceres, MT, Brasil.

5. Faculdade de Ciêncías do Desporto e Educação Física, Universidade de Coimbra, Coimbra, Portugal.

\section{Correspondência:}

Humberto Moreira Carvalho.

Faculdade de Educação Física,

Universidade Estadual de Campinas.

Av. Érico Veríssimo, 701, Campinas, SP, Brasil.13083-851.

hmoreiracarvalho@gmail.com

\section{RESUMO}

Introdução: A prática de basquetebol durante a adolescência aumenta o tamanho corporal e o desempenho funcional. O basquetebol envolve esforços máximos de curta duração com mudanças de direção que utilizam o metabolismo anaeróbico e a agilidade. Objetivo: Examinar a variação do desempenho no Line-drill Test em adolescentes jogadores de basquetebol ( $n=59$, faixa etária 9 a 15 anos) com relação ao estado de maturação somática (idade estimada do pico da velocidade de crescimento [PVC]) e à variação do tamanho corporal. Métodos: Foi considerada a idade cronológica, o tempo previsto para a idade do PVC pelo protocolo maturity offset, a estatura e a massa corporal medidas por antropometria e o desempenho no Line-drill Test. Foram utilizados modelos alométricos proporcionais para controlar a variação associada ao tamanho corporal, idade cronológica e estado de maturação na variação interindividual no Line-drill Test. Resultados: Observou-se uma relação linear negativa elevada entre o desempenho no Line-drill Test e a idade cronológica ( $r=-0,64, \mathrm{IC} 95 \%-0,77$ a -0,46, $p<0,01)$. A relação entre o desempenho no Line-drill Test e o tempo até a idade do PVC mostrou ser não linear, indicando desaceleração na variação do desempenho, aproximadamente entre a idade do PVC e um ano após o PVC predito. Os modelos alométricos mostraram uma relação negativa entre o tamanho corporal e o desempenho no Line-drill Test. A amplitude da variância explicada nos modelos alométricos proporcionais foi de 0,28 a 0,48 . Foram observados expoentes negativos de pequena magnitude para a idade cronológica e para o indicador de maturação, sendo, porém, significativos e de amplitude reduzida (-0,05 a -0,06). Conclusão: A utilização de modelos alométricos para controlar a influência do tamanho corporal tem o potencial de ajudar a compreender o desenvolvimento dos desempenhos de agilidade durante a adolescência em jogadores de basquetebol.

Descritores: atletas; basquetebol; desempenho atlético.

\section{ABSTRACT}

Introduction: The practice of basketball during adolescence increases the body size and functional performance. Basketball involves short-time maximum effort for changes of direction, which use anaerobic metabolism and agility. Objective: To examine the variation in performance in the Line-drill Test in adolescent basketball players ( $n=59$, age 9-15years) in relation to the stage of somatic maturity (estimated age of peak growth velocity [PGV]) and the variation in body size. Methods: We considered chronological age, estimated time for the age of PGV by the maturity offset protocol, height and body mass measured by anthropometry, and performance in the Line Drill Test. Proportional allometric models were used to control the variation associated with the body size, chronological age, and maturity of inter-individual variation in the Line-drill Test. Results: We found a high negative linear relationship between performance in the Line-drill Test and chronological age $(r=-0.64,95 \% / C-0.77--0.46, p<0.01)$. The relationship between performance on the Line-drill Test and the time until the age of $P G V$ proved to be non-linear, indicating slowdown in performance variation, approximately from the age of PGV and one year after the predicted $P G V$. The allometric models showed a negative relationship between body size and performance in the Line-drill Test. The amplitude of variance explained in the proportional allometric models ranged from 0.28 to 0.48 . Negative exponents of small magnitude were observed for chronological age and the indicator of maturity being, however, significant and with reduced amplitude (-0.05 to -0.06) Conclusion: The use of allometric models to control the influence of body size can potentially help to understand the development of agility performances in adolescent basketball players.

Keywords: athletes; basketball; athletic performance.

\section{RESUMEN}

Introducción: La práctica de baloncesto durante la adolescencia aumenta el tamaño corporal y el rendimiento funcional. El baloncesto implica un esfuerzo máximo de corta duración con cambios de dirección que utilizan el metabolismo anaeróbico y la agilidad. Objetivo: Examinar la variación de rendimiento en el Line-drill Testenjugadores adolescentes de baloncesto ( $n=59$, edad 9-15 años) en respecto al estado de maduración somática (edad estimada del pico de velocidad de crecimiento [PVC]) y la variación del tamaño corporal. Métodos: Se consideró edad cronológica, el tiempo estimado para la edad del PVC por el protocolo maturity offset, la estatura y la masa corporal medida por antropometría y el rendimiento en el Line-drill Test. Modelos alométricos proporcionales fueron utilizados para controlar la variación asociada con el tamaño corporal, la edad cronológica yel estado de maduración en la variación interindividual en el Line-drill Test. Resultados: Hubo una alta relación lineal negativa entre el rendimiento en el Line-drill Testy la edad cronológica ( $r=-0,64$, IC de 95\% -0,77 a-0,46, p <0,01). La relación entre el rendimiento en el Line-drill Test y el tiempo hasta la edad del PVC 
resultó ser no lineal, lo que indica una desaceleración en la variación del rendimiento, aproximadamente entre la edad del PVC y un año después del PVC previsto. Los modelos alométricos mostraron una relación negativa entre el tamaño corporal y el rendimiento en el Line-drill Test. La amplitud de la variación explicada en los modelos alométricos proporcionales fue de 0,28 a 0,48. Fueron observados exponentes negativos de pequeña magnitud para la edad cronológicay para el indicador de maduración, siendo, sin embargo, significativos y de amplitud reducida $(-0,05$ a -0,06). Conclusión: El uso de modelos alométricos para controlar la influencia del tamaño corporal tiene el potencial para ayudar a entender el desarrollo del rendimiento de agilidad durante la adolescencia en jugadores de baloncesto.

Descriptores: atletas; baloncesto; rendimiento atlético.

\section{INTRODUÇÃO}

A prática de Basquetebol demanda esforços de curta duração, alta intensidade e rápidas mudanças de direção, sendo determinantes para o melhor desempenho da modalidade ${ }^{1,2}$. Portanto, as ações cujo metabolismo anaeróbico é determinante são muito relevantes para o desempenho no jogo ${ }^{3}$. Com a sua evolução, e a maior capacidade de força dos jogadores, o jogo se torna cada vez mais veloz, sendo, portanto, a velocidade e agilidade fatores muito relevantes para o desempenho do atleta praticante do basquetebol.

Agilidade é uma habilidade multifatorial que combina aceleração, potência e reação, definida como o movimento de todo o corpo com mudança de direção e de velocidade em resposta a um estímulo ${ }^{4}$. Consequentemente, a capacidade de mudar de direção rapidamente (i.e. agilidade) é um pré-requisito para o melhor desempenho dentro do esporte ${ }^{5}$. Para que se avalie essa habilidade do atleta é necessário o uso de testes que envolvam o maior número de mudanças de direção em intervalos curtos de tempo, que simulem uma situação próxima à especificidade do jogo 4 . Particularmente, no basquetebol servir-nos-ia como exemplo a prova Line-drill Test, que se baseia num exercício comumente utilizado em treinamento de condicionamento anaeróbico de praticantes da modalidade. O teste revelou potencial discriminatório para distinguir jogadores adolescentes de basquetebol por nível de desempenho na modalidade (nacional vs local) e mostrou elevada confiabilidade e validade ${ }^{6}$.

Para avaliação da agilidade do atleta deverão ser considerados os fatores biológicos (como amorfologia do membro inferior, o percentual de gordura e o centro de massa), que influem diretamente no desempenho. Quando é considerado o contexto de jovens atletasdentro de uma modalidade esportiva, como no caso do basquetebol, estes tendem a apresentar uma homogeneidade na experiência esportiva, capacidades funcionais e habilidades específicas, no entanto a variação associada ao tamanho corporal e ao estado de maturação biológica pode ser considerável.

Os desempenhos máximos de curta duração, dependentes majoritariamente do metabolismo anaeróbico estão relacionados com o tamanho corporal (massa muscular ativa, arquitetura muscular, tipologia muscular) ${ }^{8}$. Durante o período de desenvolvimento pubertário, onde geralmente é feita a predição para a excelência esportiva ${ }^{9}$, particularmente no basquetebol onde a estatura é uma variável muito relevante. Observações em jogadores de basquetebol adolescentes destacam a importância de se considerar a variação associada à maturação e tamanho corporal nos desempenhos máximos em atletas adolescentes ${ }^{10,11}$. No entanto, informação sobre a possível influência da variação associada à maturação biológica e tamanho corporal sobre desempenhos máximos de curta duração que envolva a agilidade é ainda limitada.

O estudo da influência do tamanho corporal sobre desempenhos funcionais requer a consideração de abordagens alométricas ${ }^{10,12,13}$ para evitar correlações espúrias ${ }^{14}$ e interpretações potencialmente enviesadas sobre os desempenhos dos jovens atletas. Deste modo, o objetivo do presente estudo foi estudar a variabilidade do desempenho de agilidade alinhada pela idade estimada de pico de velocidade de crescimento na puberdade em jovens jogadores adolescentes de basquetebol. Especificamente, as diferenças interindividuais foram avaliadas considerando as alterações nas dimensões corporais associadas ao crescimento, adotando modelos alométricos proporcionais ${ }^{13}$

\section{MÉTODOS}

O presente estudo incluiu 59 atletas do sexo masculino, praticantes de basquetebol de nove a15 anos (12,88 11,64 anos). Os atletas estão envolvidos em processos formais de treinamento participando em competições oficiais promovidas pela Federação Paulista de Basketball (FPB). Os jovens atletas foram informados que sua participação era voluntária e atletas e pais ou responsáveis legais assinaram Termo de Consentimento Livre e Esclarecido. O estudo foi aprovado pelo conselho de ética local (CAAE: 49143515.3.0000.5404).

Antes das avaliações, os participantes foram instruídos a não ingerirem bebidas que contivessem cafeína pelo menos $8 \mathrm{~h}$ antes da sessão. Os testes foram realizados à mesma hora do dia (15 h às $18 \mathrm{~h}$ ). Os atletas utilizaram roupa e calçado semelhante durante as sessões de teste.

A idade cronológica dos jogadores foi considerada o valor mais próximo a 0,1 anos subtraindo-se a data de nascimento da data do teste. A estatura e altura sentado foram avaliadas com um estadiômetro portátil (Seca model 206; Seca. Hanover, MD, USA) no valor mais próximo de $0,1 \mathrm{~cm}$. O comprimento do membro inferior (subisquial) foi estimado subtraindo a altura sentado à estatura. A massa corporal foi medida com uma balança portátil (Seca model 770; Seca Hanover, MD, USA) no valor mais próximo a $0,1 \mathrm{~kg}$. As estimativas de confiabilidade do observador em relação às medidas antropométricas foram publicadas anteriormente ${ }^{6}$.

A idade no pico de velocidade de crescimento (PVC) foi estimada através do protocolo do maturity offset ${ }^{15}$. Esta técnica estima a distância, em anos, antes ou após o pico de velocidade de crescimento, através da idade cronológica, estatura, altura sentado e comprimento dos membros inferiores em cm, massa corporal em kg pela seguinte equação:

Maturity Offset $=-9,236+(0,0002708 \cdot($ comprimento do membro inferior $\cdot$ altura sentado $))+(-0,001663 \cdot($ idade $\cdot$ comprimento do membro inferior $))+(0,007216 \cdot($ idade $\cdot$ altura sentado $))+(0,02292 \cdot(($ massa corporal / estatura) $\cdot 100)$ ).

Valores negativos indicam tempo antes do PVC e valores positivos indicam tempo após o PVC. Os valores negativos foram somados e os valores positivos subtraidos à idade cronológica para estimar a idade no PVC.

A avaliação do desempenho de agilidade foi efetuada através da prova Line-drill Test. No protocolo os atletas correram o mais rápido possível num percurso vai-e-vem progressivo de 5,8 m; 14m;22,2m e 28 m, perfazendo um total de um total $140 \mathrm{~m}$ em um campo regular de basquetebol. Os participantes começavam $1 \mathrm{~m}$ da linha de fundo de uma quadra regular de basquetebol, onde estava colocada uma câmera filmadora (Sony, Tokyo, Japan) alinhada com a linha de fundo utilizada como referência para o momento inicial e final de recolha de tempo. O tempo foi registrado através 
da análise das sequências de filme registradas pela câmera colocada na linha final onde os atletas mudavam de direção na corrida vai-e-vem. A taxa de registro de imagens foi de 120 imagens por segundo e a análise do tempo foi efetuada através do software de análise de imagem disponível online e de acesso livre, Kinovea - 0.8.15 (http://www.kinovea.org). Foi providenciado encorajamento verbal para a realização de esforço máximo durante aprova. A confiabilidade da utilização de imagem para determinação do tempo de desempenho foi efetuada através da análise de concordância de 25 desempenhos de tempo em que se utilizou em simultâneo o registro de imagem pela câmera filmadora e células fotoelétricas (Speed Test 6.0 Standard, Cefise, Nova Odessa, SP, Brasil).Na análise da concordância entre os dois métodos não se observaram tendências de erros sistemáticos nem de erros proporcionais na análise da equação de calibração entre os dois métodos $(Y=0,034+0,997 \cdot X)$, com um erro de estimativa de 0,12 (95\% IC 0,10 a 0,17), e observando-se uma correlação perfeita entre os métodos. Os resultados indicam que a análise de imagem apresenta um grau de confiabilidade elevado e aceitável tendo como referência a cronometragem por células fotoelétricas.

\section{Análise estatística}

A estatística descritiva para a idade cronológica, dimensões antropométricas, idade no PVC estimada e desempenho de agilidade foi sumariado para a totalidade da amostra. A assunção de normalidade foi efetuada através de inspeção visual.

Inicialmente foram utilizados modelos alométricos simples para explorar as relações do desempenho na prova Line-drill Test com as dimensões corporais:

$$
Y=a \cdot X^{b} \cdot \varepsilon,(1)
$$

onde a corresponde ao coeficiente da intercepção com o eixo $Y, X$ corresponde à variável de tamanho corporal (geralmente a massa corporal), e b corresponde ao expoente dimensional (estatura, massa). Os valores de $a$ e $b$ foram estimados a partir de regressões lineares de transformações logaritmicas naturais das variáveis dependente e independente:

$$
\log Y=\log a+b \cdot \log X+\log \varepsilon_{,}(2)
$$

onde $Y$ representa a variável dependente (Line-drill Test) transformada (logaritmo natural, i.e., Log Line-drill Test) e X representa a variável independente transformada (Log variável de dimensão corporal).A validade dos modelos alométricos foi verificada através da verificação da função resultante $Y / X^{b}$ produzir uma variável de desempenho de agilidade independente do tamanho corporal, i.e., um valor de desempenho que controla apropriadamente para o tamanho corporal, não apresentando qualquer correlação espúria com a variável de tamanho corporal $^{16}$. Adicionalmente, foi verificada a normalidade da distribuição dos resíduos resultantes dos modelos alométricos.

Em seguida foram utilizados modelos alométricos proporcionais ${ }^{10,13}$ para incorporar o indicador de maturação biológica, adicionando-se um termo exponencial ao modelo alométrico simples (equação 1) de modo a acomodar tanto variável de dimensão corporal e a idade (centrada ao PVC) para modelar o desempenho no Line-drill Test:

\section{Line-drill Test $=$}

dimensão corporal $b \cdot \exp (c+d \cdot$ maturity offset $) \cdot \varepsilon$. (3)
O modelo poderá ser linearizado através de transformações logarítmicas naturais, onde a equação 3 se torna:

\section{$\log ($ Line-drill Test $)=$
$b \cdot \log ($ dimensão corporal $)+c+d \cdot($ maturity offset $)+\log \varepsilon .(4)$}

A associação entre os resíduos de cada modelo alométrico proporcional e o respectivo denominador alométrico foram calculados recorrendo à correlação de Pearson para verificar a assunção de independência da função de potência resultante em relação ao indicador de maturação e à respectiva variável de dimensão corporal. Adicionalmente foi verificada a assunção de homogeneidade dos resíduos dos modelos alométricos proporcionais. Os modelos alométricos consideram-se válidos para controlar os efeitos do tamanho corporal e maturação somática se a correlação entre os resíduos dos modelos e o indicador de maturação e a respectiva variável de dimensão corporal, separadamente, se aproximar de zero ${ }^{12}$. Os parâmetros desconhecidos e os respectivos intervalos de confiança a $95 \%$ nos modelos alométricos foram estimados pelo método de máxima verossimilhança. Os modelos alométricos foram obtidos recorrendo às funções "Im" $\mathrm{e}^{\text {"I }} \mathrm{me}$ " incluidas no pacote " $n / m e^{\prime \prime 17}$, estando o pacote disponível na linguagem estatística R (http://cran.r-project.org).

\section{RESULTADOS}

As características da amostra total de jogadores adolescentes de basquetebol estão apresentadas na Tabela 1. Vinte e dois participantes apresentavam valores de offset positivos e 37 apresentavam valores negativos indicando que a variabilidade interindividual no estado de maturação somática era substancial na amostra do presente estudo.

A Figura 1 ilustra as relações entre o desempenho no Line-drill Test com a idade cronológica (Painel A) e com a distância à idade do PVC (Painel B). A dispersão indica uma relação linear negativa elevada entre o desempenho no Line-drill Test e a idade cronológica ( $r=-0,64,95 \%$ IC - 0,77 a -0,46, $p<0,01)$. No entanto, namodelação da relação entre o desempenho no Line-drill Test e a distância à idade do PVC observou-se, através de comparação de modelos pelos critérios de informação de Akaike e critério de informação bayesiano, que a relação foi não linear, indicando uma desaceleração na variação no desempenho, aproximadamente, entre a idade do PVC e um ano após o PVC predito.

Os expoentes alométricos da relação entre o desempenho Line-drill Test e o tamanho corporal (equação 1) estão apresentados nos modelos I (estatura) e IV (massa corporal) da Tabela 2. Os modelos mostraram que uma relação negativa entre o tamanho corporal e o desempenho Line-drill Test, indicando que os jogadores com maior tamanho apresentaram valores superiores nos desempenhos da prova de agilidade (i.e., menor tempo na prova corresponde a um desempenho superior). Os resultados dos modelos proporcionais alométricos (equação 3) que consideram a influência da idade cronológica e da

\begin{tabular}{|c|c|c|c|}
\hline & Média & Desvio padrão & Amplitude \\
\hline Idadecronológica (anos) & 12,88 & 1,64 & $9,43-15,51$ \\
\hline Idade estimada no PVC (anos) & 13,46 & 0,60 & $11,69-14,74$ \\
\hline $\begin{array}{c}\text { Distância à idade no PVC } \\
\text { (maturity offset) (ano) }\end{array}$ & $-0,59$ & 1,63 & $-3,23-2,45$ \\
\hline Estatura $(\mathrm{cm})$ & 166,7 & 14,5 & $138,9-205,0$ \\
\hline Massa corporal (kg) & 59,4 & 17,0 & $32,4-94,7$ \\
\hline Altura sentado $(\mathrm{kg})$ & 82,8 & 7,0 & $71,4-97,3$ \\
\hline $\begin{array}{l}\text { Comprimento do membro } \\
\text { inferior }(\mathrm{cm})\end{array}$ & 83,9 & 8,5 & $66,2-111,0$ \\
\hline Line-Drill Test, (segundos) & 35,30 & 3,26 & $29,89-45,08$ \\
\hline
\end{tabular}

Tabela 1. Estatística descritiva para a amostra total $(n=59)$. 
distância à IPVC, separadamente para cada uma das dimensões corporais, estão também sumariados na Tabela 2. A amplitude da variância explicada dos modelos alométricos proporcionais foi de 0,28 a 0,48. A amplitude dos intervalos de confiança a 95\% dos expoentes alométricos sugere que a magnitude dos expoentes nos modelos proporcionais foi similar comparando com os modelos alométricos simples, no entanto o sentido da relação inverteu-se em ambas as variáveis de tamanho corporal. Os expoentes negativos para a idade cronológica e para o indicador de maturação foram de magnitude pequena, mas significativa e de amplitude reduzida $(-0,05$ a 0,06).

A análise dos resíduos resultantes dos modelos alométricos simples e proporcionais mostrou correlações de magnitude zero com as variáveis independentes em cada modelo.

\section{DISCUSSÃO}

O presente estudo investigou a variabilidade do desempenho de agilidade alinhada pela idade cronológica e pela idade estimada de PVC na puberdade em jovens jogadores adolescentes de basquetebol, adotando uma abordagem alométrica. Os modelos alométricos demonstraram validade para modelar as relações entre as dimensões corporais (estatura, massa corporal) e o desempenho no Line-drill Test, controlando adicionalmente a influência da idade cronológica e da idade estimada de PVC na puberdade. Os resultados indicaram a presençade variabilidade interindividual significativa nos desempenhos do Line-drill Test durante o período de crescimento pubertário, mesmo após o controle apropriado da influência do tamanho corporal.

Os expoentes alométricos substancialmente diferentes de 1,0 indicam que a modelação alométrica foi estatisticamente ajustadas para controlar a variação associada ao tamanho corporal. Deste modo, a utilização de rácios simples para tentar controlar a influência do tamanho corporal em provas de desempenhos máximos com corrida potencialmente produzirá correlações espúrias que poderão comprometer as interpretações. Os estudos utilizando abordagens alométricas em

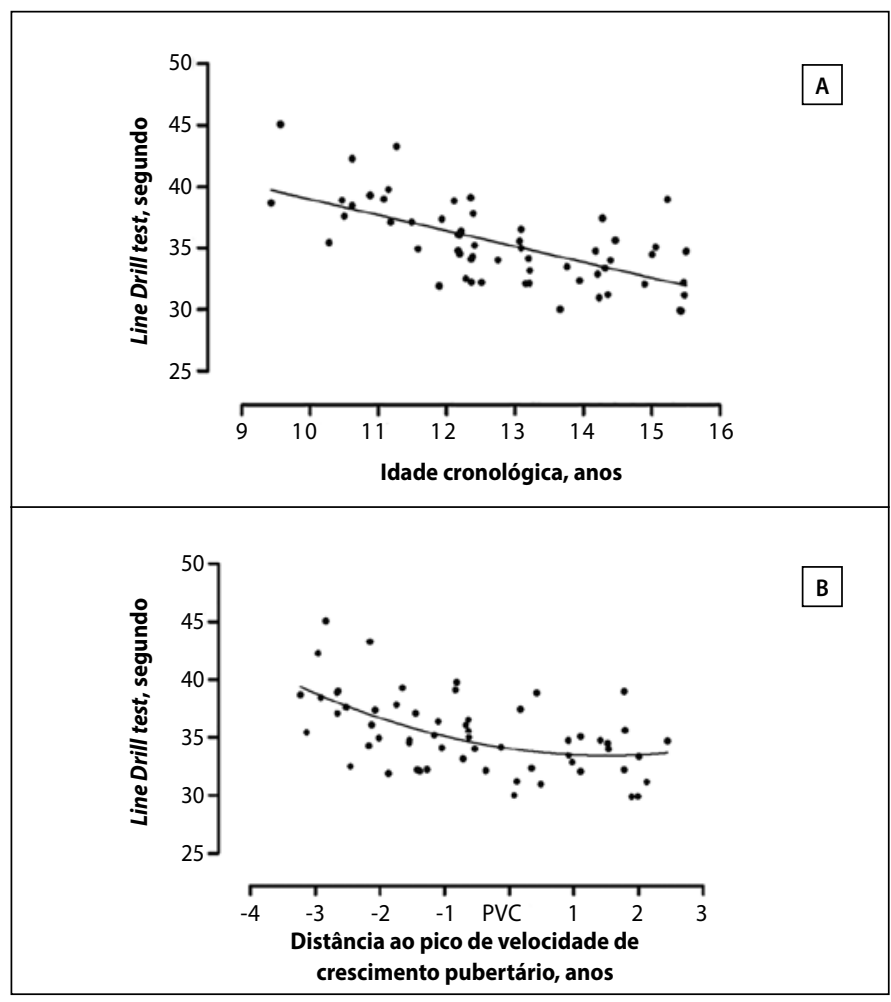

Figura 1. Desempenho na prova Line-drill Test alinhado pela idade cronológica (A) e pela idade estimada no pico de velocidade de crescimento pubertário (B) em jogadores de basquetebol adolescentes. desepenhos motores máximos em provas de corrida são limitados na literatura, particularmente em jovens atletas ${ }^{18-20}$. Em provas máximas de corrida linear em futebolistas adolescentes a amplitude dos expoentes reportada é de 0,22 - 0,66 e 0,08-0,33 para a estatura e massa corporal, respectivamente ${ }^{19,20}$. Os expoentes alométricos no presente estudo são consistentes com a amplitude de valores reportados. O sentido negativo dos expoentes alométricos no presente estudo justifica-se pela medida temporal de desempenho em que menor tempo na prova corresponde a um desempenho superior. A interpretação da magnitude e direção dos expoentes sugere que os jogadores com maiores dimensões corporais apresentaram valores superiores nos desempenhos da prova Line-dril Test.

A variação da distância estimada ao PVC e consequentemente a estimativa da idade para o PVC na presente amostra foi relativamente pequena e comparável com a variação reportada em estudos com amostras transversais de jogadores adolescentes de basquetebo ${ }^{10}$ e em estudos longitudinais que determinaram a idade no PVC ${ }^{21-23}$. Apesar de terem sido apontadas fragilidade às equações de estimativa da idade para o PVC na puberdade 24,25 , as limitações do método são evidentes, sobretudo, em crianças e adolescentes em idades mais distantes do PVC. No presente estudo 22 jogadores apresentaram valores de offset positivos e 37 apresentaram valores negativos indicando que a variabilidade interindividual no estado de maturação somática era substancial. Consequentemente, as possíveis limitações do método de estimativa da idade no PVC na amostra de jogadores adolescentes de basquetebol deverão ser consideradas e sugere precaução na interpretação e generalização dos resultados.

A variação no desempenho de agilidade mostrou-se muito relacionada com a idade cronológica. Adicionalmente, observou-se uma tendência de relação não linear entre a variação no desempenho do Line-drill Test com a idade estimada de PVC na puberdade, onde uma estabilização da variação no desempenho, aproximadamente, entre a idade do PVC e um ano após o PVC. Considerando diferenças metodológicas, os resultados do presente estudo são consistentes com observações longitudinais em

Tabela 2. Modelação alométrica do desempenho na prova Line-Drill Test controlando o tamanho corporal alinhados pela idade cronológica e pela idade estimada no pico de velocidade de crescimento (PVC) pubertário em jogadores de basquetebol adolescentes.

\begin{tabular}{|c|c|c|c|c|c|}
\hline Modelo & $\begin{array}{c}\text { Variável } \\
\text { independente }\end{array}$ & $\begin{array}{c}\text { Expoentes } \\
\text { (95\% intervalo } \\
\text { de confiança) }\end{array}$ & $P$ & $\mathbf{R}^{2}$ & $\begin{array}{c}\mathbf{R}^{2} \\
\text { ajustado }\end{array}$ \\
\hline I & Estatura & $\begin{array}{c}-0,39 \\
(-0,64 \mid-0,14) \\
\end{array}$ & 0,00 & 0,14 & 0,13 \\
\hline \multirow[t]{3}{*}{ II } & & & & 0,45 & 0,43 \\
\hline & Estatura & $\begin{array}{c}0,28 \\
(-0,02 \mid 0,53)\end{array}$ & 0,08 & & \\
\hline & $\begin{array}{c}\text { Idade } \\
\text { cronológica }\end{array}$ & $\begin{array}{c}-0,05 \\
(-0,06 \mid-0,03)\end{array}$ & 0,00 & & \\
\hline \multirow[t]{3}{*}{ III } & & & & 0,30 & 0,28 \\
\hline & Estatura & $\begin{array}{c}0,49 \\
(-0,04 \mid 1,02) \\
\end{array}$ & 0,07 & & \\
\hline & $\begin{array}{c}\text { Distância ao PVC } \\
\text { (maturity offset) }\end{array}$ & $\begin{array}{c}-0,05 \\
(-0,08 \mid-0,02)\end{array}$ & 0,00 & & \\
\hline IV & Massa corporal & $\begin{array}{c}-0,10 \\
(-0,17 \mid-0,02) \\
\end{array}$ & 0,02 & 0,10 & 0,08 \\
\hline \multirow[t]{3}{*}{ V } & & & & 0,46 & 0,44 \\
\hline & Massa corporal & $\begin{array}{c}0,09 \\
(0,01 \mid 0,17)\end{array}$ & 0,04 & & \\
\hline & $\begin{array}{c}\text { Idade } \\
\text { cronológica }\end{array}$ & $\begin{array}{c}-0,05 \\
(-0,06 \mid-0,03)\end{array}$ & 0,00 & & \\
\hline \multirow[t]{3}{*}{$\mathrm{VI}$} & & & & 0,35 & 0,32 \\
\hline & Massa corporal & $\begin{array}{c}0,19 \\
(0,05 \mid 0,32)\end{array}$ & 0,00 & & \\
\hline & $\begin{array}{c}\text { Distância ao PVC } \\
\text { (maturity offset) }\end{array}$ & $\begin{array}{c}-0,06 \\
(-0,08 \mid-0,03)\end{array}$ & 0,00 & & \\
\hline
\end{tabular}


desempenhos de agilidade em jovens futebolistas ${ }^{21} \mathrm{e} \mathrm{em}$ jovens adolescentes não praticantes de esporte ${ }^{22,26}$. A estabilização da variação nos desempenhos no Line-drill Test pode estar associada aos assincronismos nos incrementos pubertários de estatura, massa corporal, segmentos corporais e volumes musculares regionais dos membros inferiores ${ }^{27}$, contribuindo para um fenômeno descrito como "adolescent akwardeness" onde desajustes de coordenação motora estão associados aos períodos de crescimento pubertário. Consistente com as observações precedentes, os resultados dos modelos alométricos proporcionais evidenciaram um efeito independente do indicador de maturação, explicando e invertendo o sentido dos expoentes alométricos de tamanho corporal.

Por outro lado será necessário considerar que a duração do esforço no Line-drill Test implica solicitações metabólicas que envolvem, pelo menos em parte, a produção anaeróbica de ATP pela degradação de PCr e glicólise anaeróbica ${ }^{28}$. A relevância do metabolismo anaeróbico no Line-drill Testfoi evidente na associação observada com uma prova anaeróbica de 30 s em cicloergómetro em jogadores de basquetebol adolescentes ${ }^{6}$. As observações longitudinais em adolescentes sugerem que os incrementos de desempenhos anaeróbicos na adolescência estarão largamente associados ao aumento de massa muscular associados ao crescimento pubertário ${ }^{29,30}$. Deste modo, a interpretação dos desempenhos de mudança de direção no Line-drill Test deverão considerar a variação associada à idade e às mudanças qualitativas e quantitativas na massa muscular ${ }^{11,31}$ e, possivelmente, às adaptações específicas do treinamento no basquetebol. No presente estudo não foi possível considerar o controle de variáveis relacionadas com a exposição ao treino dos jovens jogadores da amostra.

\section{CONCLUSÃO}

Os resultados dos modelos alométricos proporcionais sugerem que a variabilidade interindividual associada ao tamanho corporal no desempenho dos jovens jogadores de basquetebol no Line-drill Test é largamente explicada pelo aumento das dimensões corporais durante o período de crescimento pubertário. Adicionalmente, as magnitudes dos expoentes do indicador de maturação sugerem uma influência independente da maturação somática no desempenho dos jovens jogadores de basquetebol no Line-drill Test. A utilização de modelos alométricos para controlar apropriadamente a influência do tamanho corporal poderá potencialmente ajudar a compreender os mecanismos de desenvolvimento dos desempenhos de agilidade durante a adolescência em jogadores de basquetebol.

\section{AGRADECIMENTOS}

O presente estudo foi financiado parcialmente pela Fundação de Amparo à Pesquisa do Estado de São Paulo (FAPESP). HMC foi financiado pela Coordenação de Aperfeiçoamento de Pessoal de Nível Superior (CAPES) [PNPD/CAPES/2013]. Os autores agradecem a paciência e cooperação dos atletas e treinadores. Os autores agradecem e reconhecem as contribuições na recolha de dados de Larissa Breder, Luiza Cunha, Marlus Sousa, Marina Boscariol e Kelvin Sobral.

Todos os autores declararam não haver qualquer potencial conflito de interesses referente a este artigo.

CONTRIBUIÇÕES DOS AUTORES: Cada autor contribuiu individual e significativamente para o desenvolvimento do manuscrito. HMC (0000-0002-2855-0296)*, RRP (00000002-9333-9294)* e CEG (0000-0002-6687-9041)* contribuíram para o desenho conceptual do estudo. ALAS (0000-0001-6629-656X)* e HMC foram os principais contribuintes na redação do manuscrito. ALAS, TJL (0000-0002-3843-2648)*, RSR (0000-0003-0556-9151)* e HMC realizaram as coletas de dados. HMC avaliou os dados da análise estatística. CEG, RSR, RRP e HMC fizeram a revisão do manuscrito. Todos os autores reviram e aprovaram a versão final do artigo. *ORCID (Open Researcher and Contributor ID).

\section{REFERÊNCIAS}

1. McInnes SE, Carlson JS, Jones CJ, McKenna MJ. The physiological load imposed on basketball players during competition. J Sports Sci. 1995;13(5):387-97.

2. Ben Abdelkrim N, El Fazaa S, El Ati J. Time-motion analysis and physiological data of elite under-19-year-old basketball players during competition. Br J Sports Med. 2007;41(2):69-75.

3. Sallet P, Perrier D, Ferret JM, Vitelli V, Baverel G. Physiological differences in professional basketball players as a function of playing position and level of play. J Sports Med Phys Fitness. 2005;45(3):291-4.

4. Sheppard JM, Young WB. Agility literature review: classifications, training and testing. J Sports Sci. 2006;24(9):919-32

5. Brughelli M, Cronin J, Levin G, Chaouachi A. Understanding change of direction ability in sport: a review of resistance training studies. Sports Med. 2008;38(12):1045-63.

6. Carvalho HM, Coelho e Silva M, Figueiredo AJ, Goncalves CE, Castagna C, Philippaerts RM, et al. Crossvalidation and reliability of the line-drill test of anaerobic performance in basketball players $14-16$ years. J Strength Cond Res. 2011;25(4):1113-9.

7. Malina RM. Physical growth and biological maturation of young athletes. Exerc Sport Sci Rev. 1994;22:389-433.

8. Martin JC, Malina RM. Developmental variations in anaerobic performance associated with age and sex. In: Pediatric anaerobic performance, E. Van Praagh. Champaign, IL: Human Kinetics;1998:97-117.

9. Pearson DT, Naughton GA, Torode M. Predictability of physiological testing and the role of maturation in talent identification for adolescent team sports. J Sci Med Sport. 2006;9(4):277-87.

10. Carvalho HM, Coelho e Silva MJ, Goncalves CE, Philippaerts RM, Castagna C, Malina RM. Age-related variation of anaerobic power after controlling for size and maturation in adolescent basketball players. Ann Hum Biol. 2011;38(6):721-7.

11. Carvalho HM, Coelho e Silva MJ, Figueiredo AJ, Goncalves CE, Philippaerts RM, Castagna C, et al. Predictors of maximal short-term power outputs in basketball players 14-16 years. Eur J Appl Physiol. 2011;111(5):789-96

12. Nevill AM, Ramsbottom R, Williams C. Scaling physiological measurements for individuals of different body size. Eur J Appl Physiol Occup Physiol. 1992;65(2):110-7.

13. Nevill AM, Holder RL. Modelling maximum oxygen uptake-a case-study in non-linear regression model formulation and comparison. J Royal Statistical Society, Series C (Applied Statistics). 1994;43(4):653-66.

14. Tanner JM. Fallacy of per-weight and per-surface area standards, and their relation to spurious correlation. J Appl Physiol. 1949;2(1):1-15.

15. Mirwald RL, Baxter-Jones AD, Bailey DA, Beunen GP. An assessment of maturity from anthropometric measurements. Med Sci Sports Exerc. 2002;34(4):689-94
16. Albrecht GH, Gelvin BR, Hartman SE. Ratios as a size adjustment in morphometrics. Am J Phys Anthrop. 1993;91(4):441-68.

17. Pinheiro JC, Bates DM. Mixed-effects models with Sand and S-plus. New York: Springer. 2000

18. Markovic G, Jaric S. Movement performance and body size: the relationship for different groups of tests. Eur J Appl Physiol. 2004;92(1-2):139-49.

19. Buchheit M, Mendez-Villanueva A, Mayer N, Jullien H, Marles A, Bosquet L, et al. Locomotor performance in highly-trained young soccer players: does body size always matter? Int J Sports Med. 2014;35(6):494-504.

20. Mendez-Villanueva A, Buchheit M, Kuitunen S, Poon TK, Simpson B, Peltola E. Is the relationship between sprinting and maximal aerobic speeds in young soccer players affected by maturation? Pediatr Exerc Sci. 2010;22(4):497-510.

21. Philippaerts RM, Vaeyens R, Janssens M, Van Renterghem B, Matthys $D, C$ raen R, et al. The relationship between peak height velocity and physical performance in youth soccer players. J Sports Sci. 2006;24(3):221-30.

22. Yagüe PH, De La Fuente JM. Changes in height and motor performance relative to peak height velocity: A mixed-longitudinal study of Spanish boys and girls. AmJ Human Biol. 1998;10(5):647-60.

23. Malina RM, Bouchard C, Beunen G. Human growth: selected aspects of current research on well-nourished children. Ann Rev of Anthropol. 1988;17:187-219.

24. Moore SA, McKay HA, Macdonald H, Nettlefold L, Baxter-Jones AD, Cameron N, et al. Enhancing a somatic maturity prediction model. Med Sci Sports Exerc. 2015;47(8):1755-64.

25. Malina RM, Koziel SM. Validation of maturity offset in a longitudinal sample of Polish boys. J Sports Sci. 2014;32(5):424-37.

26. Beunen G, Malina RM, Lefevre J, Claessens AL, Renson R, Simons J. Adolescent growth and motor performance: A longitudinal study of Belgian boys. Champaign, IL: Human Kinetics; 1988.

27. Beunen G, Malina RM. Growth and physical performance relative to the timing of the adolescent spurt. Exerc Sport Sci Rev. 1988;16:503-40

28. Dawson B, Goodman C, Lawrence S, Preen D, Polglaze T, Fitzsimons M, et al. Muscle phosphocreatine repletion following single and repeated short sprint efforts. Scand J Med Sci Sports. 1997;7(4):206-13.

29. Carron AV, Bailey DA. Strength development in boys from 10 through 16 years. Monogr Soc Res Child Dev. 1974;39(4):1-37

30. rmstrong N, Welsman JR, Chia MY. Short term power output in relation to growth and maturation. Br J Sports Med. 2001;35(2):118-24

31. Van Praagh E, Dore E. Short-term muscle power during growth and maturation. Sports Med. 2002;32(11):701-28 